\title{
Second-Harmonic Generation Studies of Inhomogeneous Magnetization Distributions in Thin Garnet Film
}

\author{
A. BONDA*, S. UBA AND L. UBA \\ Institute of Computer Science, University of Bialystok, Lipowa 41, PL-15424 Białystok, Poland
}

\begin{abstract}
The magnetization processes can be effectively studied using magnetization-induced second-harmonic generation effect (MSHG). The aim of the current work is the investigation of inhomogeneous magnetization distributions induced in the surface area of the garnet film by ion implantation. The studies were performed on magnetic garnet film of (111) symmetry, implanted with $\mathrm{H}_{2}^{+}$ions of $1.5 \times 10^{16} \mathrm{~cm}^{-2}$ dose and $60 \mathrm{keV}$ energy. The measurements of MSHG effect were performed as a function of the sample rotation angle and the amplitude of external magnetic field. The observed MSHG intensity hystereses were subjected to a decomposition procedure into contributions having different magnetic field dependences. The contributions originating from the implanted and unimplanted parts of the film volume of different magnetic ordering were analyzed and discussed.
\end{abstract}

DOI: $10.12693 /$ APhysPolA.127.511

PACS: 78.20.Ls, 42.65.Ky, 75.50.Gg, 75.70.-i

\section{Introduction}

Conventional experimental techniques based on magneto-optical phenomena, linear in magnetization, such as Faraday and Kerr effects are well known and widely used in studies properties of magnetic materials. Rapid laser technology development ensured to obtain strong laser pulses which an interaction with the matter induces the new nonlinear phenomena. In the past decade $[1,2]$ experimental techniques employing nonlinear optical and magneto-optical effects have been developed, providing to obtain interesting information unavailable with methods based on linear effects. These effects, occurring under high electromagnetic field intensities interacting with the matter, have been successfully applied in studies of a variety of complex systems.

In nonlinear optics, second-order effects, in particular second-harmonic generation (SHG) phenomenon, in electric-dipole approximation are only allowed in medium with broken space inversion symmetry [3]. Such a symmetry reduction generally occurs in technological processes, associated with crystals growth, and is characteristic for thin films and multilayer interfaces. Thus SHG effect constitutes sensitive and volume selective method for surfaces and interfaces investigations [4-6]. Moreover, in magnetic materials additionally to SHG effect, magnetization-induced second-harmonic generation (MSHG) effect can occur simultaneously.

In this paper we report the results of magnetic properties investigation, with the use of MSHG effect, in magnetic garnet films which the surface volume was implanted with $\mathrm{H}_{2}^{+}$ions. The measurements of MSHG intensities were performed as a function of the in-sampleplane external magnetic field $H_{\|}$and azimuthal sam-

* corresponding author; e-mail: a.bonda@uwb.edu.pl ple orientation $\varphi$. We propose simple phenomenological model which allows to perform decomposition of the observed MSHG field dependences into contributions related to different sample volumes of distinct magnetic properties.

\section{Experimental details}

In the present study the $(\mathrm{YSmLuCa})_{3}(\mathrm{FeGe})_{5} \mathrm{O}_{12}$ garnet film of the thickness of $950 \mathrm{~nm}$ grown by liquid phase epitaxy on (111)-oriented $\mathrm{Gd}_{3} \mathrm{Ga}_{5} \mathrm{O}_{12}$ substrate was implanted with $\mathrm{H}_{2}^{+}$ions of $1.5 \times 10^{16} \mathrm{~cm}^{-2}$ dose and $60 \mathrm{keV}$ energy. It is known that implantation modifies magnetic properties such as magnetization and anisotropy in a thin surface layer. The projected range of implantation is of the order of $\approx 300 \mathrm{~nm}$.

The measurements of second-harmonic intensity $I_{2 \omega}$ in MSHG effect were performed as a function of both the $H_{\|}$amplitude up to $\pm 2 \mathrm{kOe}$, and the azimuth $\varphi$ in the range of $0^{\circ}-360^{\circ}$ with respect to the incident light polarization plane. These measurements were conducted in transmission geometry with the use of the experimental setup which was described in detail in recent papers $[7,8]$. As a light source, laser system, generating femtosecond pulses at wavelength $800 \mathrm{~nm}$, was used. The $I_{2 \omega}$ intensity was measured with the photon-counting technique. The azimuthal position of the sample was controlled with the use of motorized rotation stage. The MSHG signal of $I_{2 \omega}(H, \varphi)$ at $400 \mathrm{~nm}$ was registered for the linear polarization of incident and generated waves along $X$ axis of laboratory frame. External magnetic field $H_{\|}$was applied in the sample plane along $Y$ axis.

\section{Results and discussion}

Representative results of the $I_{2 \omega}(H)$ intensity measurements for the investigated implanted garnet film for two selected azimuths $\varphi=25^{\circ}$ and $\varphi=55^{\circ}$ are shown in Fig. 1a and b, respectively. Since the azimuth $\varphi=25^{\circ}$ is close to the minimum and azimuth $\varphi=55^{\circ}$ is near 


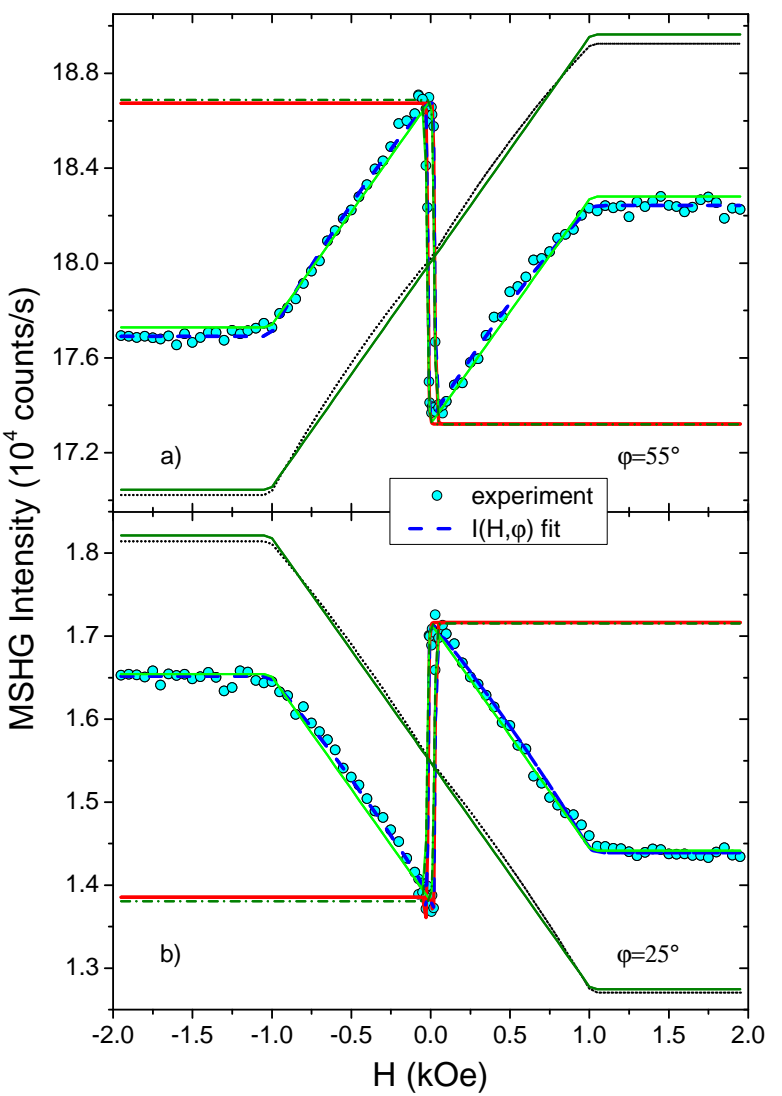

Fig. 1. Decomposition of the measured MSHG intensity vs $H_{\|}$(circles) into contributions (solid lines) originated from the implanted (s-shaped curves) and unimplanted (trapeze-shaped curves) part of the garnet film, as a result of the fit (solid lines) of the model (see text). Dotted, dashed, and dash-dotted lines correspond to the decomposition into contributions including quadratic terms in magnetizations.

to the next-neighbor maximum of the lobe, in observed rotational anisotropy $I_{2 \omega}(\varphi)$ experiments [9], the measured dependence of $I_{2 \omega}(H, \varphi)$ in Fig. 1a has a reverse dependence in comparison to the one shown in Fig. 1b, as well as over one order of magnitude smaller amplitude. Measured dependences show, at low fields, almost rectangular hystereses of a large amplitude and the coercivity of about 20 Oe, and at higher fields almost linear relationship of $I_{2 \omega}(H)$ up to the saturation at $H_{\|} \approx 1 \mathrm{kOe}$. It should be noted that the $I_{2 \omega}(H)$ dependences for the implanted garnet film are completely different in shape, in comparison to the unimplanted film studied previously in the Ref. [7].

In the phenomenological approach, the nonlinear effects of the second-order in the electric field $\boldsymbol{E}$, are described by the electric polarization $\boldsymbol{P}_{2 \omega}$, which for $i$-th component of the polarization vector $(i=x, y, z)$ is of the form $P_{2 \omega, i}(\boldsymbol{M})=\chi_{i j k}^{[2]}(\boldsymbol{M}) E_{j}(\omega) E_{k}(\omega)$, where $\chi_{i j k}^{[2]}$ is the optical susceptibility tensor, which depends on the magnetization $\boldsymbol{M}$. Expanding $\chi_{i j k}^{[2]}$ in a power series of $\boldsymbol{M}$, one can obtain an expression for $P_{2 \omega, i}(\boldsymbol{M})$, which depends explicitly on successive powers of magnetization [7]. The measured quantity in the MSHG effect is the second-harmonic light intensity, described by the expression $I_{2 \omega}=\boldsymbol{P}_{2 \omega} \cdot \boldsymbol{P}_{2 \omega}^{\star}$.

In this paper we present a phenomenological model which allows to describe the MSHG effect as a function of the $H_{\|}$amplitude in the studied implanted garnet film. The crystal structure and magnetic properties of the sample, modified by ion implantation in the surface sub-layer of garnet film, are complex and theoretical model of the system requires the adoption of simplifying assumptions. It was assumed that the implanted film is composed of two sub-layers with different effective properties. In the model, the assumption was made that $P_{2 \omega, i}$ (for $i=X$ ) of the implanted film can be expressed as the sum $P=\sum_{k} P_{k}$ of polarizations $P_{k}=a_{k}+b_{k} M_{k}$ for two sub-layers. The subscript $k=1,2$ refers to the polarization $P_{k}$ of each volume and the coefficients $a_{k}$ and $b_{k}$ in the polarization expansion which is limited to the crystallographic contribution and contributions linear in magnetization $M_{k}$. Substituting the expressions for $P_{k}$ in the total $P$ into the formula for $I_{2 \omega}$ one obtains $I_{2 \omega}=A+B_{1} M_{1}+B_{2} M_{2}+C_{1} M_{1}^{2}+C_{2} M_{2}^{2}+D M_{1} M_{2}$. The coefficients $A, B_{k}, C_{k}$ and $D$, being combinations of coefficients $a_{k}$ and $b_{k}$, are parameters of the model fit to the experimental data.

Analyzing the shape of the experimental $I_{2 \omega}(H)$ dependences (see Fig. 1) it can be seen that there is a relationship $I_{2 \omega}(-H)=-I_{2 \omega}(H)$ relative to the average signal value. This indicates that the dominant contribution to the $I_{2 \omega}$ originates from the linear components of the magnetization. Therefore, in the fitting of the theoretical $I_{2 \omega}(H)$ dependences to the experimental data, in the first approximation, only linear contributions have been considered, i.e., the dependences of $I_{2 \omega}(H)=A+B_{1} M_{1}+B_{2} M_{2}$ with three independent parameters: $A, B_{1}$ and $B_{2}$ were fitted. This is equivalent to perform the decomposition of $I_{2 \omega}(H)$ into the contributions, originating from the unimplanted and implanted film volumes, which are proportional to the $M_{1}(H)$ and $M_{2}(H)$ effective magnetizations, respectively.

It was assumed that measured $I_{2 \omega}(H)$ dependences (see Fig. 1) are the sum of two magnetic contributions. The first one is described by normalized function $M_{1}(H)$ of the specified form: $\left(H / H^{\text {sat }}\right)$ for $|H|<H^{\text {sat }}$ and \pm 1 for $|H| \geq H^{\text {sat }}$, where $H^{\text {sat }}$ is the saturation field. Provided relation describes the remagnetization process in film with perpendicular magnetic anisotropy, which can be associated with the unimplanted film volume of $H^{\text {sat }} \approx 1 \mathrm{kOe}$. The remaining contribution is described by normalized sigmoidal function $M_{2}(H)$ of the form [10]: $M_{2}^{ \pm}(H)=-1+2 /\left(1+\exp \left(-s\left(H \mp H_{c}\right)\right)\right)$, where $s$ is the softness and $H_{c}$ is the coercivity of the implanted part.

Quantitative decomposition of $I_{2 \omega}(H)$ was performed with the use of numerical fitting procedure, with $A, B_{1}$ and $B_{2}$ fitting parameters, while $H^{\text {sat }}, s$ and $H_{c}$ were the fitting parameter for the functions $M_{1}(H)$ and $M_{2}(H)$. The results of fitting the model to the experimental data 


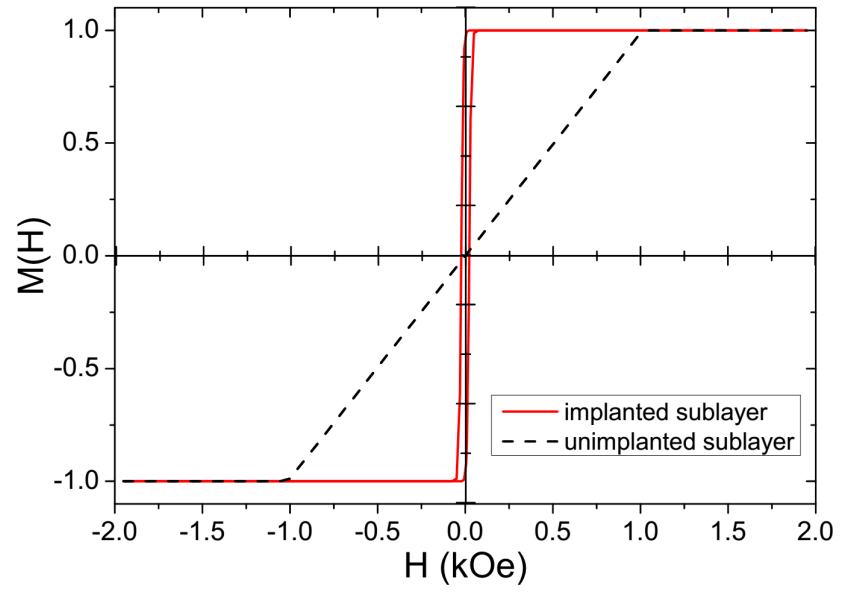

Fig. 2. Fitted magnetic field dependences of normalized magnetizations of the implanted (solid line) and unimplanted (dashed line) parts of the garnet film (see text).

of $I_{2 \omega}(H)$ are shown with solid lines in Fig. 1, and as one can see good agreement between theoretical functions and experiment has been obtained. In addition, the results of decomposition of measured $I_{2 \omega}(H)$ dependences into contributions determined for the both of considered film volumes have been shown in Fig. 1.

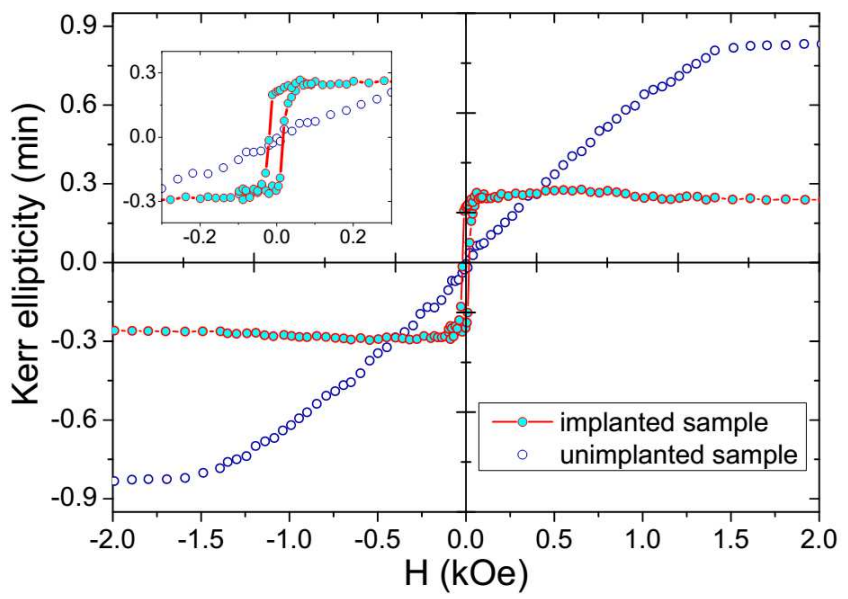

Fig. 3. Measured magneto-optical Kerr ellipticity as a function of the magnetic field $H_{\|}$for both implanted and unimplanted garnet films.

In Fig. 2, best fits to measured $I_{2 \omega}(H)$ of model prediction for normalized $M_{1}(H)$ and $M_{2}(H)$ functions have been shown. The $M_{2}(H)$ has a form of magnetic hysteresis with following parameters: $s=0.18 \pm 0.01$ and coercivity field $H_{c}=22 \pm 1 \mathrm{Oe}$, while the $M_{1}(H)$ function exhibits a trapeze-shape dependence with saturation field parameter $H^{\text {sat }}=1010 \pm 5$ Oe. Obtained $H^{\text {sat }}$ value is about 300 Oe smaller than corresponding value for the case of unimplanted film [7]. Decrease of $H^{\text {sat }}$ value in unimplanted part of the film can be related to the interaction between both film volumes of the sample.

The correctness of the obtained model results is confirmed by linear Kerr effect measurements, which reflect the remagnetization processes in implanted and unimplanted garnet samples, as shown in Fig. 3. Minor discrepancies between theory and experiment, seen in Fig. 1, can have their source in assumed model approximation, which takes into account only linear in magnetization terms of both sub-layers to the total intensity of MSHG effect. Additional fitting of the model to the experiment data was performed, where also quadratic in magnetization terms have been accounted in $I_{2 \omega}$ expression. The results are shown in Fig. 1 by dashed, dotted and dashdotted lines. As seen in Fig. 1, the quality of the fit is significantly improved and excellent agreement of theory and experiment has been obtained, where contributions from both of the sample volumes have been insignificantly only changed.

It should be concluded that for investigated garnet film, characterized by different types of effective magnetic anisotropy of implanted and unimplanted film volumes, it is possible to perform decomposition of measured intensity of MSHG effect into contributions, linear in magnetization (in first approximation) of both parts of the same sample. It should be noted, that the contributions of separated film volumes have the opposite signs in $I_{2 \omega}$, but the signs of magnetization are the same. More detailed analysis including complete MSHG azimuthal dependences will be the subject of further studies. It should be emphasized the advantage of experimental technique with the use of MSHG effect, which allows in single experiment to obtain information achievable in linear Kerr effect but for two different samples.

\section{Acknowledgments}

One of the authors (A.B.) acknowledges financial support through the project no. WND-POKL.08.02.0220-002/12 co-financed by European Social Funds, PLGovernment and Podlaskie Voivodeship.

\section{References}

[1] O.A. Aktsipetrov, J. Opt. Soc. Am. B 28, A27 (2011).

[2] Y.R. Shen, J. Opt. Soc. Am. B 28, A56 (2011).

[3] Y.R. Shen, The Principles of Nonlinear Optics, Wiley, New York 1984.

[4] H. Wierenga, W. de Jong, M. Prins, T. Rasing, R. Vollmer, A. Kirilyuk, H. Schwabe, J. Kirschner, Surf. Sci. 331-333, Part B, 1294 (1995).

[5] R. Vollmer, A. Kirilyuk, H. Schwabe, J. Kirschner, H. Wierenga, W. de Jong, T. Rasing, J. Magn. Magn. Mater. 148, 295 (1995).

[6] F. Hansteen, O. Hunderi, T.H. Johansen, A. Kirilyuk, T. Rasing, Phys. Rev. B 70, 094408 (2004).

[7] A. Bonda, S. Uba, L. Uba, Phys. Rev. B 87, 024426 (2013).

[8] A. Bonda, S. Uba, L. Uba, Acta Phys. Pol. A 121, 1225 (2012)

[9] V.N. Gridnev, V.V. Pavlov, R.V. Pisarev, A. Kirilyuk, T. Rasing, Phys. Rev. B 63, 184407 (2001).

[10] J.F. McGilp, L. Carroll, K. Fleischer, J. Phys.: Condens. Matter 19, 396002 (2007). 